\title{
Searching for new physics in bottomonium decays
}

\section{Miguel Sanchis-Lozano*i}

IFIC-Departamento de Fisica Teorica, Valencia University, Spain

E-mail: Miguel.Angel.Sanchis@uv.es

\begin{abstract}
Heavy quarkonium decays can be used to search for New Physics beyond the Standard Model. In particular, a light Higgs boson could induce a slight (but observable) lepton universality breaking in Upsilon leptonic decays. In fact, current experimental data from CLEO presented in this Conference seem to point out to this direction within experimental accuracy. Moreover, LEP constraints on a light Higgs mass can be evaded by different models (like MSSM with a CPV Higgs sector) as shown in this Conference. We also consider spectroscopic consequences stemming from a possible mixing between Higgs and bottomonium states leading to discrepancies with the SM expectations (e.g. hyperfine splittings).
\end{abstract}

International Europhysics Conference on High Energy Physics

July 21 st - 27th 2005

Lisboa, Portugal

\footnotetext{
${ }^{*}$ Speaker.

${ }^{\dagger}$ Research under grant FPA2002-00612, GV-GRUPOS03/094 and GV05/276.
} 


\section{Introduction}

The CLEO collaboration has recently released experimental results on muonic branching fractions (BF's) of Upsilon resonances below open bottom threshold [1]. Moreover, CLEO has presented in this Conference new and more precise results on the tauonic BF's of all three $\Upsilon(1 S)$, $\Upsilon(2 S)$ and $\Upsilon(3 S)$ states, the latter representing the first ever observed tauonic decay rate of this resonance. By combining these new data with the already available PDG values [2], we show in Table 1 the current (though preliminary) status of all six BF's, thereby allowing a test of lepton universality in Upsilon decays. Deviation from this hypothesis can be assessed using the ratio

$$
R_{\tau / \ell}(n S)=\frac{\mathscr{B}[\Upsilon(n S) \rightarrow \tau \tau]-\mathscr{B}[\Upsilon(n S) \rightarrow \ell \ell]}{\mathscr{B}[\Upsilon(n S) \rightarrow \ell \ell]} \quad, \quad \ell=e, \mu
$$

As pointed out in the literature $[3,4,5,6,7]$, lepton universality breaking in Upsilon decays would open up the possibility of New Physics (NP) beyond the Standard Model (SM), indicating the existence of a light non-standard Higgs boson. Such a particle would mediate the $b \bar{b}$ annihilation into a tauonic pair subsequent to a dipole magnetic (either allowed or hindered) transition of the Upsilon, yielding a soft (unobserved) photon according to the cascade process

$$
\Upsilon(n S) \rightarrow \gamma_{s} \eta_{b}\left(n^{\prime} S\right)\left(\rightarrow \tau^{+} \tau^{-}\right) \quad ; \quad n \geq n^{\prime}=1,2,3
$$

In our later development, we shall factorize the above cascade decay as

$$
\mathscr{B}\left[\Upsilon(n S) \rightarrow \gamma_{s} \tau^{+} \tau^{-}\right]=\mathscr{B}\left[\Upsilon(n S) \rightarrow \gamma_{s} \eta_{b}\left(n^{\prime} S\right)\right] \times \mathscr{B}\left[\eta_{b}\left(n^{\prime} S\right) \rightarrow \tau^{+} \tau^{-}\right]
$$

Because the photon would escape unnoticed ${ }^{1}$, such a NP contribution would be unwittingly ascribed to the tauonic decay mode, while the electronic or muonic modes would result unaltered, ultimately implying lepton universality breaking.

From a theoretical viewpoint, the existence of a light pseudoscalar in the Higgs sector is compatible with certain extensions of the SM involving two Higgs doublets [8], e.g. a Peccei-Quinn symmetry can yield a pseudo-Nambu-Goldstone boson, which for a range of model parameters (for example, in the NMSSM) is significantly lighter than the other scalars.

\section{Experimental results}

In figure 1 we plot the values of $R_{\tau / \ell}(n S)$ stemming from the results shown in Table 1. According to a hypothesis test (where lepton universality plays the role of the null hypothesis) lepton universality can be rejected at the $1 \%$ level of significance.

On the other hand, let us note the fact that no $\eta_{b}$ resonance has been found so far despite intensive searches along many years. Recently CLEO has also made a search for the $\eta_{b}(1 S)$ and $\eta_{b}(2 S)$ states via hindered magnetic dipole transitions from $\Upsilon(3 S)$ with negative results too [9]. In fact, one might speculate that this failure is due to quite broad ${ }^{1} S_{0}$ bottomonium states as a consequence of the new physics contribution [3,5]. Mixing between a CP-odd Higgs (denoted as $A^{0}$ ) and pseudoscalar bottomonium states may also lead to $\Upsilon-\eta_{b}$ hyperfine splittings different from the SM expectations [3]

\footnotetext{
${ }^{1}$ As far as it is not searched for; it should be possible, however, to look for such soft photons in the tauonic subsample of CLEO on-tape recorded events
} 
Table 1: Measured leptonic branching fractions $\mathscr{B}[\Upsilon(n S) \rightarrow \ell \ell]$ (in \%) and error bars (summed in quadrature) of $\Upsilon(1 S), \Upsilon(2 S)$ and $\Upsilon(3 S)$ resonances (obtained from CLEO data given at this Conference and [2]).

\begin{tabular}{ccccc}
\hline channel: & $e^{+} e^{-}$ & $\mu^{+} \mu^{-}$ & $\tau^{+} \tau^{-}$ & $R_{\tau / \ell}(n S)$ \\
\hline$\Upsilon(1 S)$ & $2.38 \pm 0.11$ & & $2.66 \pm 0.11$ & $0.12 \pm 0.06$ \\
\hline$\Upsilon(1 S)$ & & $2.48 \pm 0.06$ & $2.66 \pm 0.11$ & $0.07 \pm 0.05$ \\
\hline$\Upsilon(2 S)$ & $1.92 \pm 0.17$ & & $2.03 \pm 0.28$ & $0.06 \pm 0.15$ \\
\hline$\Upsilon(2 S)$ & & $1.93 \pm 0.17$ & $2.03 \pm 0.28$ & $0.05 \pm 0.15$ \\
\hline$\Upsilon(3 S)$ & $1.92 \pm 0.24$ & & $2.51 \pm 0.26$ & $0.31 \pm 0.17$ \\
\hline$\Upsilon(3 S)$ & & $2.18 \pm 0.21$ & $2.51 \pm 0.26$ & $0.15 \pm 0.15$ \\
\hline
\end{tabular}

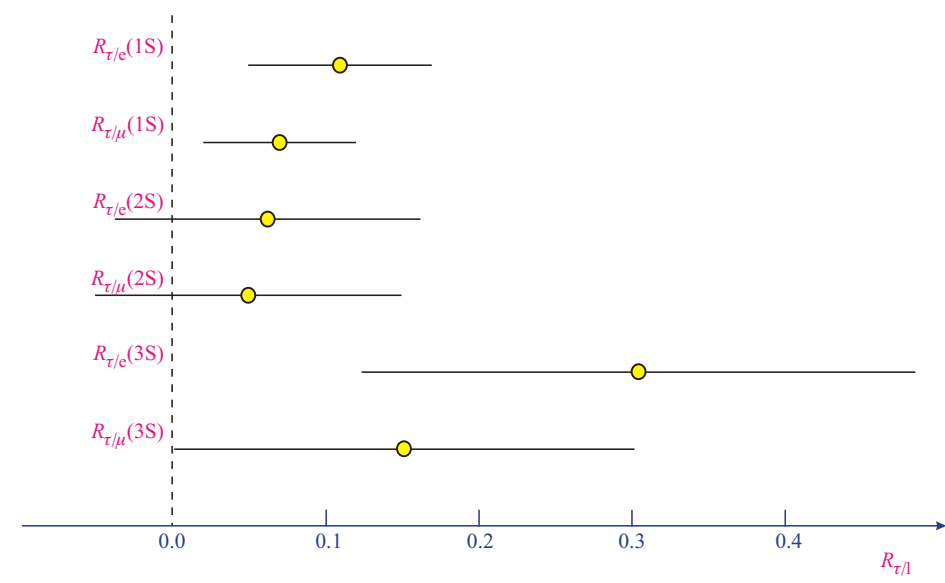

Figure 1: Plot of $R_{\tau / \ell}$ values corresponding to Table 1. Errors bars (partly due to systematic errors) are expected to be reduced after completion of the CLEO on-going analysis.

For moderate values of $\tan \beta$ (defined as the two Higgs vacuum expectation values in a 2HDM) the $\eta_{b}$ decay would be almost saturated by the Higgs-mediated annihilation into a tau pair, according to the expression for the width [6]

$$
\Gamma\left[\eta_{b} \rightarrow \tau^{+} \tau^{-}\right]=\frac{3 m_{b}^{4} m_{\tau}^{2}\left(1-4 x_{\tau}\right)^{1 / 2}\left|R_{n}(0)\right|^{2} \tan ^{4} \beta}{2 \pi^{2}\left(m_{\eta_{b}}^{2}-m_{A^{0}}^{2}\right)^{2} v^{4}}
$$

where $x_{\tau}=m_{\tau}^{2} / m_{\Upsilon}^{2}, R_{n}(0)$ stands for the radial $\eta_{b}$ wave function at the origin and $v=246 \mathrm{GeV}$. Hence assuming $\mathscr{B}\left[\eta_{b} \rightarrow \tau^{+} \tau^{-}\right] \approx 1$, we can conclude from Eq.(1.2) that

$$
\mathscr{B}\left[\Upsilon(n S) \rightarrow \gamma_{s} \tau^{+} \tau^{-}\right] \approx \mathscr{B}\left[\Upsilon(n S) \rightarrow \gamma_{s} \eta_{b}\left(n^{\prime} S\right)\right]
$$

where the latter branching ratio can be estimated as a M1 transition probability given by [10]

$$
\mathscr{B}\left[\Upsilon(n S) \rightarrow \gamma_{s} \eta_{b}\left(n^{\prime} S\right)\right]=\frac{\Gamma^{M 1}}{\Gamma_{\Upsilon}}=\frac{16 \alpha}{3}\left(\frac{e_{b}^{2}}{2 m_{b}^{2}}\right) I k^{3}
$$

where $\alpha$ is the fine structure constant, $e_{b}$ and $m_{b}$ are the electric charge and mass of the bottom quark, $k$ denotes the photon energy and $I$ represents the initial and final wave functions overlap. 
Tentatively assuming universality breaking as a working hypothesis, the results shown in Table 1 (and figure 1) are compatible with the following interpretation involving new physics:

- There is a light CP-odd (or without definite CP) Higgs particle whose mass lies around the $\Upsilon(1 S)$. A M1 transition of a $\Upsilon(n S)(n=1,2,3)$ resonance would yield an intermediate $\eta_{b}(1 S)$ state, subsequently decaying via a Higgs-mediated annihilation channel into a $\tau^{+} \tau^{-}$ pair with almost unity probability - even for moderate $\tan \beta$ values

- Following the previous remark, the BF's shown in Table 1 are compatible with either an allowed transition of the $\Upsilon(1 S)$ into a $\eta_{b}(1 S)$ state, or a hindered transition from a $\Upsilon(2 S)$ or a $\Upsilon(3 S)$ into a $\eta_{b}(1 S)$ too, both with probabilities of order $10^{-4}-10^{-3}$ according to potential quark model calculations [10]. Thus, since $\mathscr{B}\left[\Upsilon(n S) \rightarrow \ell^{+} \ell^{-}\right] \simeq 2 \%$, one gets naturally

$$
R_{\tau / \ell}(n S) \approx \frac{\mathscr{B}\left[\Upsilon(n S) \rightarrow \gamma_{s} \eta_{b}(1 S)\right]}{\mathscr{B}[\Upsilon(n S) \rightarrow \ell \ell]} \approx 10^{-2}-10^{-1}
$$

- In addition, one should also consider radiative decays of $\Upsilon(2 S)$ and $\Upsilon(3 S)$ into an on-shell $A^{0}$ particle subsequently decaying into a tauonic pair [3]. Setting, e.g., $\tan \beta=15$ and $M_{\Upsilon}-$ $M_{A^{0}}=250 \mathrm{MeV}$, one obtains

$$
R_{\tau / \ell}(n S) \approx \frac{M_{\Upsilon}^{2} \tan ^{2} \beta}{8 \pi \alpha v^{2}}\left[1-\frac{M_{A^{0}}^{2}}{M_{\Upsilon}^{2}}\right] \approx 10^{-1}
$$

\section{Conclusions}

In the absence of any (unknown) systematic shift of the values of Table 1, the simplest explanation of lepton universality breaking would require a light non-standard Higgs mediating the tauonic decay mode as advocated in this paper. Further confirmation should be needed, e.g., by searching for those M1 soft photons in the collected sample of tauonic decays by CLEO. I acknowledge CLEO Collaboration for extremely valuable help when developing this work.

\section{References}

[1] G. S. Adams et al. [CLEO Collaboration], Phys. Rev. Lett. 94 (2005) 012001 [arXiv:hep-ex/0409027].

[2] S. Eidelman et al., Phys. Lett. B592, 1 (2004)

[3] M. A. Sanchis-Lozano, arXiv:hep-ph/0503266.

[4] N. Brambilla et al., arXiv:hep-ph/04012158

[5] M. A. Sanchis-Lozano, Nucl. Phys. Proc. Suppl. 142, 163 (2005) [arXiv:hep-ph/0407320].

[6] M. A. Sanchis-Lozano, Int. J. Mod. Phys. A 19, 2183 (2004) [arXiv:hep-ph/0307313].

[7] M. A. Sanchis-Lozano, Mod. Phys. Lett. A 17, 2265 (2002) [arXiv:hep-ph/0206156].

[8] J. F. Gunion, H. E. Haber, G. Kane and S. Dawson, The Higgs Hunter's Guide (Addison-Wesley Publishing Company, Redwood City, CA, 1990).

[9] M. Artuso et al. [CLEO Collaboration], Phys. Rev. Lett. 94 (2005) 032001 [arXiv:hep-ex/0411068].

[10] S. Godfrey and J. L. Rosner, Phys. Rev. D 64 (2001) 074011 [Erratum-ibid. D 65 (2002) 039901] [arXiv:hep-ph/0104253]. 